\title{
Ferritin as a Marker of Severe Dengue in Children
}

\author{
Manchala Chandra Sekhar ${ }^{\circledR}$ \\ Assistant Professor, Department of Pediatrics, Maheshwara Medical College \& Hospital, Patancheru, Medak, Telangana, India.
}

\section{Abstract}

Background: The aim is to evaluate ferritin levels in fever caused by dengue virus and their association with the severity of infection with dengue virus in children. Subjects and Methods: It is a Prospective that was done for 1 year in the department of pediatrics. 56 reported cases with a history of dengue fever for clinical evaluation by positive IgG and IgM antibodies and positive NS1 antigen and were included in the sample. All 16-year-old subjects with clinical dengue suspicion presenting in the department of emergency. Results: Out of total of 56 children with mean age of the children were 11.5 years and SD 3.5 years, and 59\% were male who were majority. Mean hospital stay duration was $5 \pm 3$ days. Most of the patients in study discharged in 5-7 days of admission in hospital. The mean serum ferritin levels among cases with DHF were raised than those with DF with significant p-values of $<0.05$ on Day 5 and 6 of illness. During Days 5, 6, and 7 after the onset of fever, the results for sensitivity were 83, 86, and 92 per cent respectively. Conclusion: The incidence of hyperferritinaemia in patients infected with dengue virus is suggestive of highly active use of ferritin level in serum is $1,200 \mathrm{ng} / \mathrm{ml}$ as a method for predicting progression to DHF starting on Day 4 of the disease and continuing to recover.

Keywords: Ferritin level, Dengue fever, convalescent stage

Corresponding Author: Manchala Chandra Sekhar, Assistant Professor, Department of Pediatrics, Maheshwara Medical College \& Hospital, Patancheru, Medak, Telangana, India.

E-mail: drmanchala@gmail.com

Received: 1 June 2020

Revised: 6 July 2020

Accepted: 14 July 2020

Published: 10 October 2020

\section{Introduction}

One of the world's leading viral hemorrhagic fevers is Dengue fever which is widely spread of insect borne viral diseases caused by the 4- serotyped Flavivirus genus Arbovirus, affecting a 39700 lakh people in 128 countries including India. This is propagated by mosquitoes like Aedes albopictus and Aedes aegypti. Early diagnosis by laboratory findings of dengue fever is important for providing proper treatment to cases and for preventing dangerous complications such as dengue shock syndrome (DSS) and hemorrhagic fever (DHF), both known by WHO as severe dengue. Widely used study involves the detection of dengue specific Immunoglobulin $\mathrm{G}$ or Immunoglbulin M by ELISA kit testing. Liver biochemistry is often usually deranged in dengue fever; SGOT and SGPT in particular are elevated. Increased serum ferritin has been associated with extreme dengue in children. ${ }^{[1-3]}$

Hyperferritinemia in dengue infected subjects is related with intemperate immune activation and coagulation disarranges as reported in Dengue episode. ${ }^{[4]}$ An Indian study found that serum ferritin levels in dengue infected cases are significantly higher compared to controls; Results from previous studies have shown that hyperferritinemia is associated with extreme disease that is observed during the duration of the disease. Besides other laboratory results in dengue fever, ferritin levels are also rised. Ferritin is an acute phase reactant and is expressed in response to infection or inflammation by cells of the reticuloendothelial system. Hyperferritinemia was found to be related with seriousness of dengue fever in young people. ${ }^{[5,6]}$

In adult patients, ferritin levels were also found to be related to viral load. These findings gave the impression that the serum ferritin levels in adult patients could also be used as an early indicator of dengue fever severity. Aim is to study serum ferritin level in dengue fever and its association with dengue virus infection severity at a tertiary center.

\section{Subjects and Methods}

This prospective observational study was done for duration of 1 year in the department of paediatrics.56 reported patients with history of dengue fever, clinical evaluation by $\mathrm{IgG}$ and IgM antibodies positive and positive NS1 antigen were included in the sample. 


\section{Inclusion criteria:}

All cases of 16 years age and older with clinical dengue suspicion presenting in emergency room.

\section{Exclusion criteria:}

Patients with breastfeeding, patients with documented hematological conditions, patients with qualitative and quantitative platelet conditions, patients with other febrile diseases and mixed infections, patients with heparin, radiotherapy, chemotherapy and patients with immunosuppression.

\section{Diagnostic Criteria}

DHF's clinical diagnosis is based on 4 main features of manifestations that the WHO recognises (1997):

- Persistent high grade fever lasting for two to seven days;

- Positive tourniquet test or petechiae with hemorrhagic tendency

- Thrombocytopenia

- Proof of plasma drain demonstrated by hemoconcentration or pleurual effusion.

The extent of DHF is graded into four grades as Grade-I, II, III and IV.

Patients without evidence of hemoconcentration had a right lateral decubitus x-ray of the chest taken 12-24 hours after defervescence. Both DHF and DF patients had infected with dengue virus was confirmed serologically.

Data were collected and analysed for all patients admitted to the medical ward and medical PICU during the period when the incidence of dengue has raised. After informed consent, $2 \mathrm{ml}$ of blood was collected from the study subjects; ferritin assay was done at the time of diagnosis if the diagnosis was made in the study centre; or at the time of admission if referred from an outside hospital with positive test results (NS1 or dengue serology positive). Patients with NS1 positivity on Days 2-8 and/or positive IgM for dengue on Days 6-10 prior to discharge were regarded to be positive for dengue cases.

Dengue Immunoglobin $\mathrm{G}$ and Immunoglobin $M$ were estimated by micro ELISA kit autoanalyzer and NS1 positivity was estimated by ELISA kit. Ferritin was estimated by ECLIA. LFT was estimated by Dry Chemistry System Hemoglobin, Total Count, Differencial Count and platelet were estimated by autoanalyzer in the lab.

This study performed descriptive statistical analysis. Continuous measurement results are based on mean $\pm \mathrm{SD}$, and categorical measurement results are reported in numbers (percent).

Significance is measured at $<0.05$. Microsoft Word 2010 and Microsoft Excel 2010 have been used to generate tables and figures.

\section{Results}

The study had included a total of 56 students. The children's mean age was 11.5 years and SD 3.5 years, with 59 percent being male. 21 children were classified as having DF and 35 as having DHF in which 15 grades I, 12 grades II and 8 grades III and IV were categorised according to the WHO criteria

Mean hospital stay duration was $5 \pm 3$ days. Most of the patients in study discharged in 5-7 days of admission in hospital.

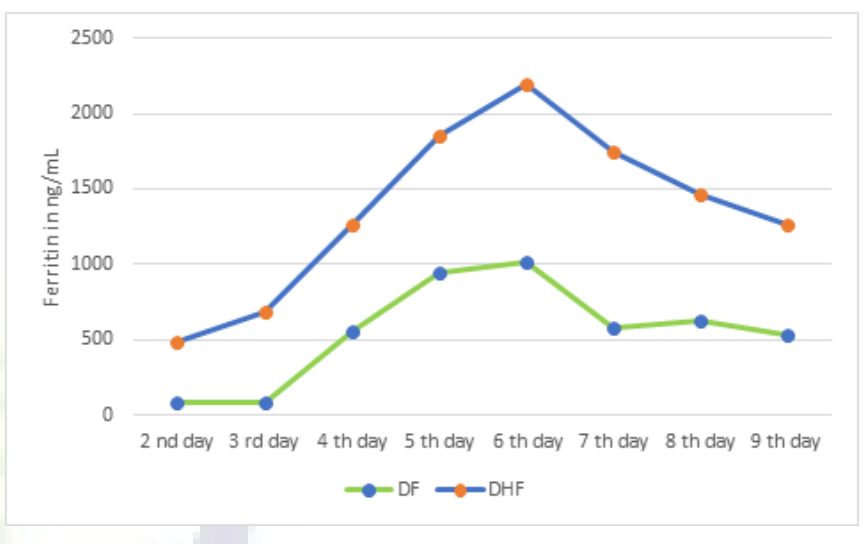

Figure 1: Ferritin

The mean serum ferritin levels among the patients with DHF were raised than those with DF with significant p-values of $<$ 0.05 on Day 5,6 of illness. At follow- up 3-4 weeks after discharge from the hospital in both the patients with DF and DHF were similar, and in the normal range.

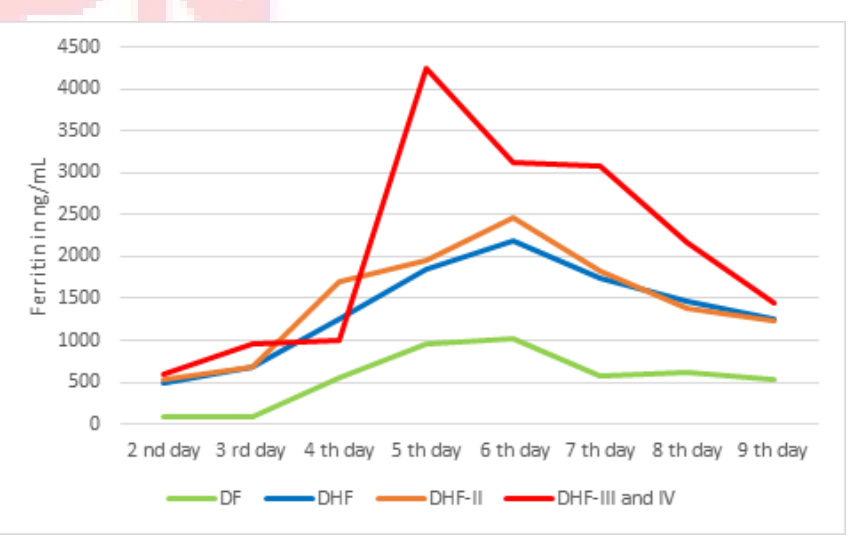

Figure 2: Comparison of mean serum ferritin levels after onset of fever between patients with DF and DHF grades per day of illness.

To predict the clinical expression of DHF, a cut-off level for ferritin in serum is of $1.200 \mathrm{ng} / \mathrm{ml}$ was used. During days 5, 
Table 1: Demographic details of study

\begin{tabular}{|c|c|c|c|c|}
\hline Diagnosis & Males & Females & Total & Mean age + SD \\
\hline & $\begin{array}{l}\text { Number of children } \\
(\%)\end{array}$ & $\begin{array}{l}\text { Number of children } \\
(\%)\end{array}$ & $\begin{array}{l}\text { Number of cases } \\
(\%)\end{array}$ & \\
\hline Dengue fever & $12(36.4 \%)$ & $9(39.1 \%)$ & $21(37.5 \%)$ & $11.2+2.6$ \\
\hline DHF grade-I & $9(27.3 \%)$ & $6(26.1 \%)$ & $15(26.8 \%)$ & $10.5+3.9$ \\
\hline DHF grade-II & $7(21.2 \%)$ & $5(21.7 \%)$ & $12(21.4 \%)$ & $12.7+4.7$ \\
\hline DHF grade-III-IV & $5(15.2 \%)$ & $3(13.1 \%)$ & $8(14.3 \%)$ & $11.7+3.1$ \\
\hline Total & $33(59 \%)$ & $23(41 \%)$ & $56(100 \%)$ & $11.5+3.5$ \\
\hline
\end{tabular}

Table 2: Duration of Hospital stay in patients

\begin{tabular}{llll}
\hline Hospital stay in days & $3-5$ days & $5-7$ days & $>7$ days \\
Males & $10(30.3 \%)$ & $20(60.6 \%)$ & $3(9.1 \%)$ \\
Females & $8(34.8 \%)$ & $14(60.9 \%)$ & $1(4.3 \%)$ \\
Total & $18(32.1 \%)$ & $34(60.7 \%)$ & $4(7.1 \%)$ \\
\hline
\end{tabular}

6 , and 7 after the onset of fever, the results for sensitivity were 83,86 , and 92 per cent respectively.

\section{Discussion}

Ferritin is an acutephase reactant, and monocytes, macrophages, and hepatic cells contain a large amount. It has been shown that cytokines and iron can induce ferritin synthesis. ${ }^{[7]} \mathrm{We}$ showed that a proinflammatory cytokine profile had been associated with increased levels of ferritin. In present study out of 56 children mean age of the children was 11.5 years and SD 3.5 years, and $59 \%$ were male the majority of the patient were male. Similar to S. Selvamuthukumaran study the majority of patients belonged to the male gender $59.19 \%(\mathrm{n}=87) .{ }^{[8]}$ A research done at Singaporerevealed that PCR + patients with fever due to dengue virus were male (71percent). ${ }^{\text {[9] }}$

Mean hospital stay duration was $5 \pm 3$ days. Most of the patients in study discharged in 5-7 days of admission in hospital. Our study is similar with Wathanee Chaiyaratana et al study of with men hospital stay of 5+2 days. Fever, the majority of the patient was also male. The clinical presentation and duration of hospital stay and seasonal variation in our study was In a Lahore based study, regarding dengue comparable with similar studies. He mean hospital stay was $2.73 \pm 1.41$ days as observed by Ahmed et al, and 3.7 \pm 1.02 days in a study conducted in Rawalpindi. ${ }^{[10]}$

In present study mean levels of serum ferritin among the patients with DHF were raised than those with DF with significant p-values of $<0.05$ on Day 5 of illness, Day 6 of illness. All these severe dengue patients had substantially raised serum ferritin levels upon admission to the hospital. ( $>500 \mathrm{ng} / \mathrm{dl}$ ). Prompted us and helped us in keeping these patients under critical hemodynamic monitoring and surveillance from day 1 of admission to our study. The same has been shown in numerous foreign studies. ${ }^{[5]}$

Another study carried out in one hundred and seventy-seven Thai children evaluating ferritin levels in serum during the clinical course showed similar findings. ${ }^{[6]}$ Increased serum ferritin was associated with severe dengue; patients with nonsevere dengue had serum ferritin levels less than $1,200 \mathrm{ng} / \mathrm{ml}$ during the entire course of illness, while severe forms recorded raised levels of ferritin in serum in the febrile stage and the defevervescence stage. Patients with dengue haemorrhagic fever (DHF) grade III and IV had significantly higher median ferritin levels measured on day 5,6 and 7 of illness than those with grade I, II DHF and dengue fever. In that study it was concluded that serum ferritin level $\geq 1,200 \mathrm{ng} / \mathrm{ml}$ from day 4 of the illness predicts the occurrence of dengue haemorrhagic fever.

In a study conducted in South India by Soundravally $\mathrm{R}$ et al, ${ }^{[11]}$ which included 48 dengue cases and 48 cases with other febrile illness as controls, serum ferritin levels were measured on the day of admission (which is a median of 4 days after the onset of fever) and day of defervescence. Compared to other febrile illnesses, dengue cases demonstrated significant increase in serum ferritin levels; also within clinical groups, severe disease exhibited higher ferritin levels compared to non-severe group throughout the febrile and defervescence stages of illness $(940.09 \pm 568.31$ in non-severe cases vs. $1264.71 \pm 492.59$ in severe dengue on day of admission; $418.19 \pm 404.59$ vs. $1490.74 \pm 359.40$ on day of defervescence) The study concluded that raised serum ferritin levels could predict the severity of dengue with a sensitivity and specificity of $76.9 \%$ and $83.3 \%$ respectively. The present study also showed similar results; hyperferritinemia was noted in severe 
cases compared to non-severe group from day 4 to day 7 of illness.

Another study showed significant association between on the day of admission raised serum ferritin levels and severe dengue development. ${ }^{[10]}$ Serum ferritin was measured on the day of admission in a total of 104 dengue positive cases and they were grouped into a) Group A with normal ferritin levels (up to $100 \mu \mathrm{g} / \mathrm{dl}$ ) and b) Group B with high serum ferritin levels; both the groups were followed up for the occurrence of severe dengue. Severe dengue was noted only in 2 out of 31 patients with normal serum ferritin levels while more than half of the patients with severe dengue (35 out of 73 ) had raised serum ferritin levels. The study concluded that serum ferritin can be used as an early marker to predict the severity of the disease.

Another significant observation made out in the present study was that age had a positive co-relation with the serum ferritin levels and the duration of hospital stay while it negatively co-related with the platelet count. This implies that age is an important predictor of severity of dengue infection. As age advances, there is increased risk of developing severe disease with low platelet count, high ferritin levels and prolonged hospital stay. Studies had shown that elderly individuals are at risk of developing severe disease. In a study conducted in Southern Taiwan during a dengue epidemic of 5336 dengue cases, the prevalence of dengue haemorrhagic fever and dengue shock syndrome were substantially higher in people 60 years of age and above compared to young subjects.$^{12}$ Coincidental findings were made in a study in Singapore; people over 60 years of age had more extreme dengue occurrence (20.3 percent vs. 14). ${ }^{[12-14]}$

Our study show 1,200 ng / ml serum ferritin cut-off level was used to predict the clinical manifestation of DHF. During Days 5,6 , and 7 after the onset of fever, the results for sensitivity were 83,86 , and 92 per cent respectively. The best cut-off level for ferritin was 1291 , with 82.6 percent sensitivity and 100 percent specificity. However this is slightly different from Ho et al. ${ }^{[15]}$ results, which registered 44 percent sensitivity and 88 percent specificity. Study done by Soundravally R et al. ${ }^{[11]}$ clinched on increased levels of ferritin could predict dengue severity with 76.9 per cent sensitivity and 83.3 per cent specificity on the day of admission. Study performed in Aruba and Brazil found that raised levels of ferritin were significantly correlated with severity of disease in dengue virus infection. ${ }^{[5]}$ Wathanee Chaiyaratana et al, ${ }^{[6]}$ research findings were 81.5 , 84.4 and 89.9 percent respectively for sensitivity during 5, 6 and 7 days of the disease after the onset of fever.

To predict progression to DHF, the cut-off level of serum ferritin is $1.200 \mathrm{ng} / \mathrm{ml}$ has an increased sensitivity but a decreased specificity. In subjects with DHF grades three and four, this could be skewed by the few cases that appeared on Days 2 or day 3 of the sickness. DHF's early diagnosis is beneficial in order to provide sufficient medical treatment for a better outcome.

\section{Conclusion}

Serum ferritin levels taken on admission can serve as a reliable and cost- effective biomarker in the assessment and in predicting the severity of dengue fever. The Serum ferritin levels can also help as an adjunct investigation in delineating the spectrum of dengue suspected patients. Serum ferritin levels may help in identifying an uncomplicated dengue illness, from that of patients who may require inpatient admission / intensive critical care . The existence of increses ferritin above normal in patients infected with dengue virus is suggestive of highly active disease which results in immune activation and coagulation disorders. Therefore we suggest close evaluation of patients with hyperferritinaemia.

The use of a serum ferritin level alone of 1,200 $\mathrm{ng} / \mathrm{ml}$ ought to be advised as a method for predicting progression to DHF starting on Day 4 of the disease and continuing to recover. We conclude therefore that the level of serum ferritin may be used to track and predict the incidence and outcome of dengue fever.

\section{References}

1. Abubakar SA, Shafee N. Outlook of dengue in Malaysia: a century later. Malays J Pathol. 2002;24(1):23-27.

2. Duong V, Lambrechts L, Paul RE, Ly S, Lay RS, Long KC. Asymptomatic humans transmit dengue virus to mosquitoes. Proc Natl Acad Sci. 2015;112(47):14688-14693. Available from: https://doi.org/10.1073/pnas.1508114112.

3. Lu P, Hsiao H, Tsai J. Dengue Virus-Associated Hemophagocytic Syndrome and Dyserythropoiesis: A Case Report. Kaohsiung J Med Sci. 2005;21(1):34-39. Available from: https: //doi.org/10.1016/S1607-551X(09)70274-8.

4. de Souza VAUF, Fernandes S, Araújo ES, Tateno AF, Oliveira OMNPF, dos Reis Oliveira R, et al. Use of an Immunoglobulin G Avidity Test To Discriminate between Primary and Secondary Dengue Virus Infections. J Clin Microbiol. 2004;42(4):1782-1784. Available from: https://dx. doi.org/10.1128/jcm.42.4.1782-1784.2004.

5. van de Weg CAM, Huits RMHG, Pannuti CS, Brouns RM, van den Berg RWA, van den Ham HJ, et al. Hyperferritinaemia in Dengue Virus Infected Patients Is Associated with Immune Activation and Coagulation Disturbances. PLoS Negl Trop Dis. 2014;8(10):e3214-e3214. Available from: https://dx.doi. org/10.1371/journal.pntd.0003214.

6. Chaiyaratana W, Chuansumrit A, Atamasirikul K, Tangnararatchakit K. Serum ferritin levels in children with dengue infection. Southeast Asian J Trop Med Public Health. 2008;39(5):832-868.

7. Sam SS, Omar SFS, Teoh BT, Abd-Jamil J, AbuBakar S. Review of Dengue Hemorrhagic Fever Fatal Cases Seen Among Adults: A Retrospective Study. PLoS Negl Trop Dis. 2013;7(5):e2194-e2194. Available from: https://dx.doi.org/10. 1371/journal.pntd.0002194. 
8. Selvamuthukumaran S. Severity of Dengue Fever and Serum Ferritin Levels- A Correlative Study in a Rural Tertiary Care Medical College and Hospital in Tamil Nadu (South India). Ann Int Med Dent Res. 2018;4(3):52-54. Available from: https://dx.doi.org/10.21276/aimdr.2018.4.3.me13.

9. Lee LK, Gan VC, Lee VJ, Tan AS, Leo YS, Lye DC. Clinical relevance and discriminatory value of elevated liver aminotransferase levels of Dengue severity. Plos Negl Trop Dis. 2012;6(6):1676. Available from: https://dx.doi.org/10. 1371/journal.pntd.0001676.

10. Ahmed A, Alvi AH, Butt A, Nawaz AA, Hanif A. Assessment of Dengue fever severity through liver function tests. J Coll Physicians Surg Pak. 2014;24:640-684. Available from: https: //doi.org/10.5772/intechopen.68949.

11. Soundravally R, Agieshkumar B, Daisy M, Sherin J, Cleetus CC. Ferritin levels predict severe dengue. Infection. 2015;43(1):13-19. Available from: https://dx.doi.org/10.1007/ s15010-014-0683-4.

12. Liu CC, Huang KJ, Huang MC, Lin JJ, Wang SM, Liu JJ. High case-fatality rate of adults with dengue hemorrhagic fever during an outbreak in non-endemic Taiwan: risk factors for dengue- infected elders. Am J Infect Dis. 2008;4:10-17. Available from: https://doi.org/10.3844/AJIDSP.2008.10.17.

13. Rowe EK, Leo YS, Wong JGX, Thein TL, Gan VC, Lee LK, et al. Challenges in Dengue Fever in the Elderly:
Atypical Presentation and Risk of Severe Dengue and HospitaAcquired Infection. PLoS Negl Trop Dis. 2014;8(4):e2777e2777. Available from: https://dx.doi.org/10.1371/journal. pntd.0002777.

14. Petchiappan V, Hussain TM, Thangavelu S. Can serum ferritin levels predict the severity of dengue early?: an observational study. J Res Med Sci. 2019;7(3):876-876. Available from: https://dx.doi.org/10.18203/2320-6012.ijrms20190940.

15. Ho T, Wang S, Anderson R, Liu C. Antibodies in dengue immunopathogenesis. J Formos Med Assoc. 2013;112(1):1-2.

Copyright: (C) the author(s), 2020. It is an open-access article distributed under the terms of the Creative Commons Attribution License (CC BY 4.0), which permits authors to retain ownership of the copyright for their content, and allow anyone to download, reuse, reprint, modify, distribute and/or copy the content as long as the original authors and source are cited.

How to cite this article: Sekhar MC. Ferritin as a Marker of Severe Dengue in Children. Asian J. Clin. Pediatr. Neonatol. 2020;8(3):7-11.

DOI: dx.doi.org/10.47009/ajcpn.2020.8.3.2

Source of Support: Nil, Conflict of Interest: None declared. 